\title{
Método simple para el cálculo de sistemas solares térmicos centralizados con acumulación estacional
}

\author{
Mateo de Guadalfajara \\ GITSE (Grupo de Ingeniería Térmica y Sistemas Energéticos) \\ Instituto de Investigación en Ingeniería de Aragón (I3A). \\ Universidad de Zaragoza, Mariano Esquillor s/n, 50018, Zaragoza, Spain. \\ Tel.+34-976762707, Fax +34-976762043, e-mail: mateog@unizar.es
}

\begin{abstract}
Para el aprovechamiento de la energía solar se han desarrollado en el norte y centro de Europa, Canada y otros países, plantas solares térmicas de baja temperatura $\left(<100^{\circ} \mathrm{C}\right)$, integradas en redes de calefacción de distrito (district heating) capaces de cubrir una parte importante de la demanda de calor (fracción solar) del sector residencial comercial [1]. El desarrollo de estos sistemas requiere de la creación de herramientas que faciliten su cálculo y diseño. Se ha desarrollado un método simple de cálculo que permite prediseñar y estimar el funcionamiento de estos sistemas en base a datos climáticos y de demanda simples, con un coste computacional sensiblemente inferior al de las herramientas de cálculo habituales.
\end{abstract}

\section{Introducción}

El consumo de energía final para la producción de calefacción y agua caliente sanitaria (ACS) del sector residencial supone un $12 \%$ del consumo nacional: transporte $40 \%$, industria $29 \%$, usos diversos 31\% (incluido calefacción y ACS) [2]. Estas necesidades pueden ser cubiertas mediante sistemas centralizados que produzcan calor de baja temperatura aprovechando recursos renovables como: biomasa, energía solar térmica, calor de cogeneración o calor residual industrial; o por el contrario, como se está haciendo ahora, consumiendo gasóleo, gas natural y electricidad de manera individual en cada edificio/vivienda. Los sistemas solares térmicos con acumulación estacional (CSHPSS Central Solar Heating Plant with Seasonal Storage) podrían aprovechar un recurso abundante en España: la radiación solar. Además estos sistemas pueden aportar nuevas opciones de gestión al sector eléctrico nacional absorbiendo la sobreproducción eléctrica acumulándola en forma de energía térmica. Esta forma de acumulación es más económica que la utilización de baterías electroquímicas o que la acumulación de hidrógeno.
Los sistemas CSHPSS presentan un comportamiento altamento dinámico en el que el funcionamiento de una instalación depende de la radiación y la demanda ambas variables en el tiempo. Debido a esto, el cálculo de estos sistemas se realiza (de forma general) mediante simulaciones dinámicas que utilizan datos horarios climáticos y de demanda de uno o varios años. En base a simulaciones con TRNSYS [3] (Transient System Simulation tool) se ha estudiado el comportamiento de estos sistemas en España [4] y se ha determinado su viabilidad y elevado potencial en nuestro país. Dada la complejidad de los cálculos se vió la necesidad de desarrollar una herramienta más simple, que permita el dimensionado rápido de los principales equipos y proporcione una estimación del resultado final.

\section{Método simple}

Se presenta un método original [5] programado en el software Engineering Equation Solver (EES), que utiliza datos climáticos públicos y fáciles de obtener. Este Método Simple permite calcular de un modo rápido y directo el funcionamiento del sistema CSHPSS en periodos mensuales y dimensionar los equipos (área del campo de captadores solares y volumen del acumulador térmico) para obtener el comportamiento deseado, evitando la simulación detallada..

En la tabla 1 se muestran los resultados mensuales para el caso de una planta situada en Zaragoza que cubre aproximadamente el $50 \%$ de la demanda de calefacción de 1000 viviendas de $100 \mathrm{~m}^{2}$. La planta tiene un campo de captadores solares, de superficie $3.293 \mathrm{~m}^{2}$ y un acumulador térmico (tanque de agua caliente) que funciona entre $30^{\circ} \mathrm{C}$ y $90^{\circ} \mathrm{C}$ de volumen $23.048 \mathrm{~m}^{3}$. Asimismo (Tabla 1), se comparan los resultados mensuales y anuales obtenidos con simulaciones dinámicas realizadas con TRNSYS. ( $Q_{c}$ producción térmica de los captadores solares; $\mathrm{Q}_{\text {solar }}$ calor solar aportado a la demanda; $\mathrm{Q}_{\mathrm{d}}$ demanda de calefacción; $\mathrm{Q}_{\mathrm{g}}$ calor auxiliar aportado) 
Tabla 1. Flujos mensuales de calor del sistema (MWh) y resultado anual (MWh/año)

\begin{tabular}{l|lll|lll|l}
\hline & \multicolumn{3}{|c|}{ Sim.TRNSYS } & \multicolumn{3}{c|}{ Método Simple } & Datos \\
\hline & $\mathrm{Q}_{\mathrm{c}}$ & $\mathrm{Q}_{\text {solar }}$ & $\mathrm{Q}_{\mathrm{g}}$ & $\mathrm{Q}_{\mathrm{c}}$ & $\mathrm{Q}_{\text {solar }}$ & $\mathrm{Q}_{\mathrm{g}}$ & $\mathrm{Q}_{\mathrm{d}}$ \\
\hline Ene. & 189 & 199 & 1110 & 185 & 185 & 1124 & 1309 \\
Feb. & 242 & 229 & 636 & 242 & 242 & 623 & 865 \\
Mar. & 333 & 319 & 313 & 322 & 322 & 310 & 632 \\
Abr. & 333 & 224 & 142 & 342 & 342 & 24 & 366 \\
May. & 374 & 80 & 0 & 403 & 80 & 0 & 80 \\
Jun. & 357 & 0 & 0 & 377 & 0 & 0 & 0 \\
Jul. & 373 & 0 & 0 & 387 & 0 & 0 & 0 \\
Ago. & 337 & 0 & 0 & 331 & 0 & 0 & 0 \\
Sep. & 158 & 0 & 0 & 206 & 0 & 0 & 0 \\
Oct. & 164 & 142 & 0 & 139 & 142 & 0 & 142 \\
Nov. & 188 & 807 & 0 & 74 & 807 & 0 & 807 \\
Dic. & 174 & 977 & 310 & 104 & 813 & 474 & 1287 \\
\hline Anual & 3224 & 2978 & 2510 & 3112 & 2933 & 2555 & 5488 \\
\hline
\end{tabular}

El método simple resulta útil para el diseño de estas instalaciones al permitir complejos análisis paramétricos. Con el método simple se ha calculado la fracción solar para múltiples combinaciones área de captadores - volumen de acumulación y mediante interpolación de datos se han obtenido las líneas isocuantas de fracción solar mostradas en la Fig. 2. Cabe decir que siguiendo la línea correspondiente a una fracción solar dada puede intercambiarse área de captadores por volumen de acumulación obteniendo la misma producción. En las zonas A y $B$ un aumento en el volumen de acumulación o en el área de captadores aumenta la fracción solar obtenida. En la zona C cualquier diseño con un volumen de acumulación menor obtendrá un mejor resultado con un menor coste. La elección del valor más adecuado de estas dos variables dependerá de los límites del proyecto y del criterio de diseño elegido.

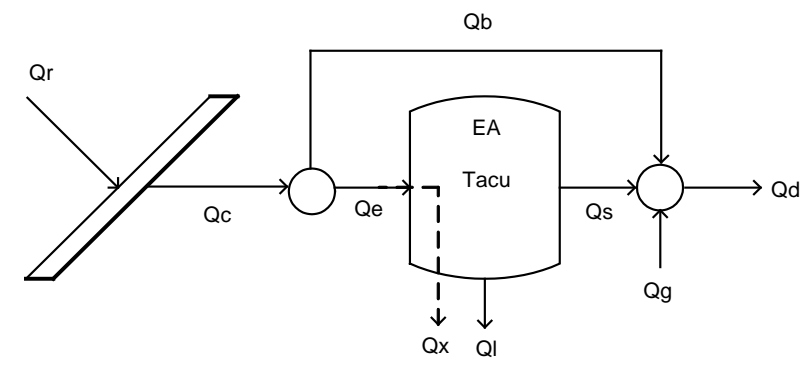

Figura 1: Diagrama de flujos del sistema
Incluyendo en el Método Simple estimaciones del coste de los equipos se pueden realizar análisis paramétricos para encontrar diseños de mínimo coste. La lína roja de la figura une diseños de mínimo coste obtenidos para distintas fracciones solares.

\section{Conclusiones}

Los sistemas CSHPSS presentan muchas ventajas estratégicas (independencia energética, acumulación de sobreproducción, utilización de recursos renovables...) y tienen un gran potencial de desarrollo en España. El método simple puede servir para facilitar la evaluación de estas instalaciónes, incluyendo factores económicos y criterios de diseño complejos. La validación de resultados del método simple no es completa todavía pero la comparación con simulaciones dinámicas indica un alto grado de similitud.

\section{REFERENCIAS}

[1] SDH. Solar Disitrict Heating. www.solar-district-heating.eu [Consulta: Abril 2013]

[2] MITyC, IDAE. Consumo de Energía Final - Informe anual de consumos energéticos. Ministerio de Industria Turismo y Comercio, IDAE, secretaría general departamento de planificación y estudios, 2010.

[3] TRNSYS 16. A Transient System Simulation tool. Solar Energy Laboratory, University of Wisconsin-Madison 2004.

[4] M. Guadalfajara, M.A. Lozano, L.M. Serra. Evaluation of the potential of large solar heating plants in Spain. Solar Heating and Cooling for Buildings and Industry (SHC 2012), Energy Procedia 2012;30:839-848.

[5] M. Guadalfajara. Evaluación de centrales solares térmicas con acumulación estacional para el sector residencia. Trabajo Fin de Máster, EINA, Universidad de Zaragoza, 2013.

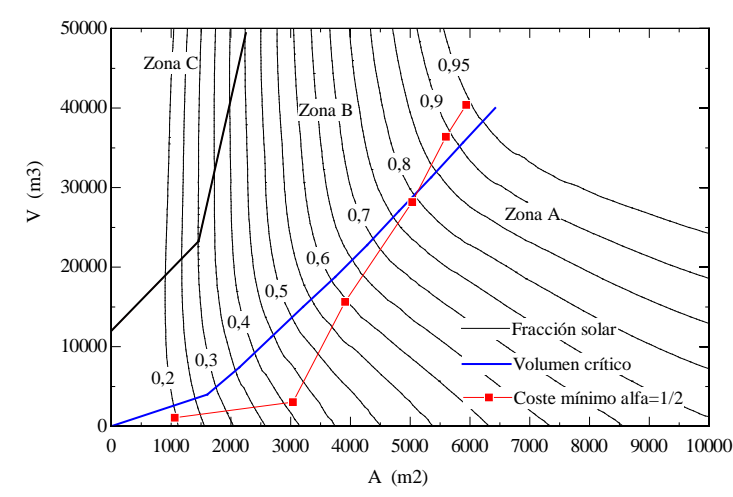

Figura 2: Criterios de diseño de CSHPSS, intercambio de variables: Área de captadores - Volumen acumulación. 